\title{
The qualitative behavior of coupled predator-prey oscillations as deduced from simple circle maps
}

\author{
John Vandermeer \\ Department of Biology, University of Michigan, Ann Arbor, MI 48109, USA
}

(Received 18 November 1992; accepted 11 June 1993)

\begin{abstract}
Qualitative patterns of the non-equilibrium behavior of coupled consumer/resource systems, formulated as a standard circle map, are explored. Patterns of demographic locking, reflected as a "devil's staircase", are observed in simulations of the MacArthur consumer/resource equations. As coupling strength increases the steps in the staircase become broader, eventually overlapping to form a chaotic pattern. In addition to the standard form of chaos formation from overlapping Arnold tongues, a pattern of period doubling in the non-invertible standard circle map corresponds to the pattern observed in the classic consumer/resource equations.
\end{abstract}

Key words: Circle map; Non-equilibrium behavior; Predator-prey interaction

\section{Introduction}

The original form of the Lokta-Volterra predator/prey equations did not include the biological force normally associated with dissipation, density dependence, and thus the equations produced behavior that was inherently oscillatory yet non-dissipative (May, 1972, 1981). Adding simple terms to account for density dependence turns the characteristic "centers" into stable foci. Furthermore, May (1981) noted that most realistic models include forces that provided for the generation of an unstable focus at some local level, which usually result in a stable limit cycle globally. It is thus normally assumed that predator/prey models will oscillate, sometimes damping to a single limit point, other times maintaining a permanent oscillation on a limit cycle, depending on the exact mix of parameter values (May, 1981; Vandermeer, 1993).

The intrinsic "consumption" of the predator in such systems implies that the predator/prey pair, or, equivalently, the consumer/resource pair, form a sort of 
structural base for ecological communities, giving underlying meaning to both the classic population interactions (parasite/host, predator/prey, competition (MacArthur, 1970; Abrams, 1980, 1986; Chesson, 1990), mutualism (Levine, 1976; Vandermeer, 1980)), and trophic levels (by definition). As such, the details of their dynamics ought to be a central goal of community ecology, as has indeed been the case (e.g. Hsu and Hubbell, 1979; Abrams, 1975, 1980, 1986; Chesson, 1990).

Recently it has been noted that when such consumer/resource pairs are coupled together (as surely they must be in any real ecological community) some unexpected patterns result, including frequency entrainment, period doubling, and chaos (Vandermeer, 1993). Given that the elementary consumer/resource system is inherently oscillatory, it makes sense to analyze a coupled pair of such systems as a coupled oscillator (Vandermeer and Kaufmann, 1994a,b). Assuming consumer / resource couplings are similar to others of nature's coupled oscillators, it is possible to borrow a powerful technique from physics, circle maps (Bohr et al., 1984; Bak, 1986; Cvitanovic et al., 1990), to gain qualitative insight into the apparently complicated behavior that may result from coupling oscillators. The purpose of this note is to explore some of the emergent qualitative patterns of non-equilibrium behavior of coupled consumer/ resource systems, using the tool of the circle map.

\section{Basic theory}

Consider population densities or biomasses of two consumers, $y_{1}$ and $y_{2}$ and two resources, $x_{1}$ and $x_{2}$, where $y_{i}, x_{i} \in \mathrm{R}^{+}$. Presume that their dynamics are stipulated by a set of ordinary autonomous differential equations, namely,

$$
\begin{array}{ll}
\mathrm{d} y_{i} / \mathrm{d} t=f_{i}\left(y_{i}, x_{i}\right), & i=1,2, \\
\mathrm{~d} x_{i} / \mathrm{d} t=g_{i}\left(y_{i}, x_{i}\right), & i=1,2 .
\end{array}
$$

Assume that $f$ and $g$ and the parameters therein are chosen in such a way that each subsystem (i.e. $x_{1}, y_{1}$ and $x_{2}, y_{2}$ ), when operating in isolation, will exhibit limit cycles. The goal is to study what happens when such isolated oscillations are coupled together.

As is standard procedure (Jackson, 1989; Arrowsmith and Place, 1990), the behavior of two oscillators together can be modelled as movement on a torroidal surface. By placing the two oscillations at right angles to one another and rotating one of the oscillators around the other, it is a simple matter to track out the relevant torus. All trajectories of the 4D system, whether coupled or independent, can be visualized as trajectories on the surface of this torus.

With two identical predator/prey systems operating independently of one another, the trajectory on the torus winds around both dimensions once. To observe the long-term behavior of the system it is convenient to construct a Poincaré section at some point and observe the "return" behavior of one consumer/ resource system as determined by the other. With two totally unconnected 
but identical systems, any arbitrary starting point winds around the torus once in both dimensions, and thus appears as a single point on the shell of the Poincare section (Abraham and Shaw, 1988).

In any natural setting it seems very unlikely that the periods of both consumer/ resource pairs are precisely the same, and indeed the situation becomes far more interesting when one period is slightly different than the other. Each cycle around the torus cuts the Poincaré section at a slightly different point. The effect is that the intersection on the Poincaré section is in a different position each cycle.

These dynamics can be conveniently represented with a simple transformation. Consider

$$
\theta=\tan ^{-1}\left[\left(C-C^{*}\right) /\left(R-R^{*}\right)\right],
$$

where the $C=$ consumer (predator), $R=$ resource (prey), and the asterisk indicates the mean value of the variable in its limit cycle. The quantity $\theta$ is thus proportional to the consumer/resource (predator/prey) ratio and $\theta$ ranges from 0 to $2 \pi$ radians.

The qualitative dynamics of the overall system can be deduced by tracing successive values of $\theta$ as they cut through the Poincaré section, the equation for the map thus derived is

$$
\theta(t+1)=\Omega+\theta(t), \bmod 1,
$$

where $\Omega$ is the winding number (the excess amount of winding of one oscillator with reference to the other). The $1 \mathrm{D}$ map described above is known as a circle map.

In the ideal situation with the oscillating frequency of both consumer/ resource pairs identical, the appropriate circle map is simply $\theta(t+1)=\theta(t)$, a simple $45^{\circ}$ line in which whatever $\theta$ is initiated on the Poincaré section, it is returned to exactly, as the trajectory strikes the Poincaré section again. If the trajectories are not independent (e.g. the first consumer eats some of the second consumer's resources), $\theta(t+1)=\theta(t)$ would no longer be true. Thus, we presume $\theta(t+1)=$ $f(\theta)$, where $f$ is some function not too different from $\theta(t)$ alone.

In Fig. 1 is the qualitative form of the map that in fact does emerge, for a wide variety of consumer/resource equations. It is clear that there are actually two equilibrium points, one stable and the other unstable, indicating that there should be two points on the Poincaré section, one of which repels trajectories, the other of which attracts them. This arrangement is sometimes referred to as a "braid", and, due to a powerful theorem known as Peixoto's theorem, all such arrangements in torroidal flow must have an equal number of stable versus unstable torroidal cycles (Abraham and Shaw, 1988).

The biological significance of these observations is simply that with a loose connection of two consumer/resource pairs, trajectories that normally cover the torus entirely, whether by never repeating themselves or by being defined uniquely, suddenly are locked together on a single stable trajectory. No matter where the system is initiated, it always winds up on that single stable trajectory. This is the 


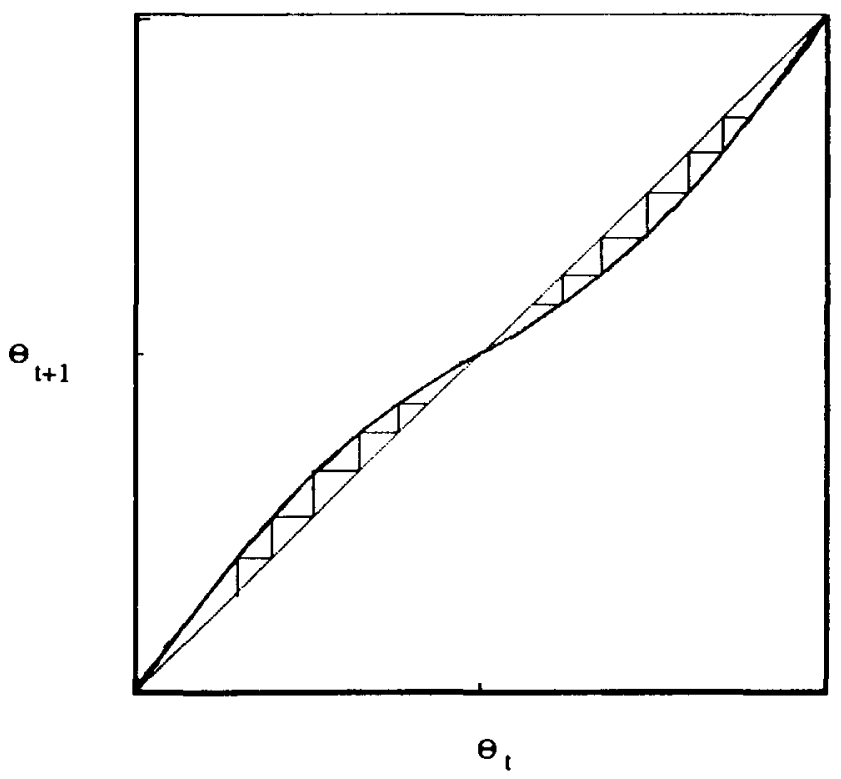

Fig. 1. Dynamics of the circle map with weakly coupled consumer resource systems with identical periodicity.

parallel result of the stable equilibrium point or stable limit cycle of the classic Lotka Volterra equations, but with a 4-dimensional system.

To complete the general theoretical framework, consider the distortion likely to be introduced to the map $\theta(t+1)=\theta(t)$ if the coupling of systems is further increased. If the map $\theta(t+1)=\theta(t)+f(\theta)+\Omega$ is to change with coupling strength, we assume the change will be in the form of smooth changes in the non-linearities involved in $f(\theta)$, that those changes in the circle map introduced through the act of coupling the independent $2 \mathrm{D}$ systems will simply be exaggerated as the coupling increases in strength.

While a great many specific forms of $f(\theta)$ could be imagined, it has become customary to examine this general phenomenon with a particularly simple form, the "standard" circle map,

$$
\theta(t+1)=\Omega+\theta(t)+(k / 2 \pi) \sin [2 \pi \theta(t)],
$$

where $k$ is taken as proportional to the coupling strength (Bohr et al., 1984; Bak, 1986). While the arguments herein do not appear to be dependent on the specific form of $f(\theta)$, it is convenient to use the standard map.

\section{Demographic locking}

Utilizing the standard circle map as a model of two consumer/resource systems, it is possible to suggest generic behaviors for such systems. In particular, if 
$p_{i}$ represents the period of the $i$ th consumer/ resource pair, we allow $\Omega=p_{i} / p_{j}$ to be the ratio of periods for the two systems operating independently of one another. We then examine the parameter,

$$
w=\lim _{n \rightarrow \infty}\left(f_{n}(\theta)-\theta\right) / n
$$

usually called the winding number, where $f_{n}$ is the standard circle map, and $n$ is the number of iterations. The well-known result is that the periods tend to lock onto one another, at rational values of $w$, or become distorted at irrational values of $w$. If $w$ is rational the point where the trajectory cuts the Poincaré section will eventually repeat itself, whereas if $w$ is irrational, the point will never repeat itself in the Poincaré section (Bohr et al., 1984).

Selecting a specific level of coupling (a fixed value of $k$ in the standard circle map) and computing $w$ for a range of values of $\Omega$, a pattern emerges in which the winding number remains at a fixed rational value over a range of values of $\Omega$, and furthermore, an infinite number of irrational winding numbers exist between rational couplings. This result has been termed the devil's staircase, since the staircase effect (fixed rational values over a range of $\Omega$, interspersed with variable irrational values) has a fractal character. If a small section of the graph of $w$ versus $\Omega$ is blown up, it also exhibits the staircase effect.

It is of some interest to examine the consumer/resource equations from this point of view, expecting the qualitative behavior predicted from the circle map will be reflected in the differential equations. Allowing $y$ to be the predator (consumer) and $x$ the prey (resource), we write,

$$
\begin{aligned}
& \mathrm{d} y_{1} / \mathrm{d} t=r_{1} y_{1}\left(1-g y_{1} /\left(1+x_{1}+c x_{2}\right)\right), \\
& \mathrm{d} y_{2} / \mathrm{d} t=r_{2} y_{2}\left(1-g y_{2} /\left(1+x_{2}+c x_{1}\right)\right), \\
& \mathrm{d} x_{1} / \mathrm{d} t=r_{3} x_{1}\left(1-x_{1} / K\right)-a x\left(y_{1}+c y_{2}\right) /\left(1+x_{1}+c x_{2}\right), \\
& \mathrm{d} x_{2} / \mathrm{d} t=r_{4} x_{2}\left(1-x_{2} / K\right)-a x\left(y_{2}+c y_{1}\right) /\left(1+x_{2}+c x_{1}\right),
\end{aligned}
$$

where $r_{i}$ is the intrinsic rate of natural increase for the $i$ th population, $K$ is the carrying capacity of the resources, $a$ and $g$ are constants, and $c$ is the coupling coefficient. Setting $c=0.01$, the empirical winding number (Eq. 1) was calculated for a range of values of $r_{4}$ from 2.0 to 6.5, with the other parameters set as follows: $r_{1}=r_{2}=0.5, r_{3}=7.0, K=20, a=0.1, g=0.04$.

As can be seen in Fig. 2, these consumer resource equations illustrate exactly the behavior predicted by the circle map, a devil's staircase, with rational locking at all the exact predicted ratios (the following ratios are clearly observable as steps in the devil's staircase: $3 / 2,4 / 3,5 / 4,7 / 5,6 / 5,9 / 7,11 / 8$, and $10 / 7$ ). There is a pattern of demographic locking that follows exactly the sequence observed in other, mainly physical, systems (Bak, 1986). While further ramifications of this observation remain under investigation, little doubt exists that these equations will likely behave similarly to the general qualitative predictions of the circle map, including the generation of Arnold tongues and chaos resulting from the interplay of competing frequencies (Bak, 1986). 

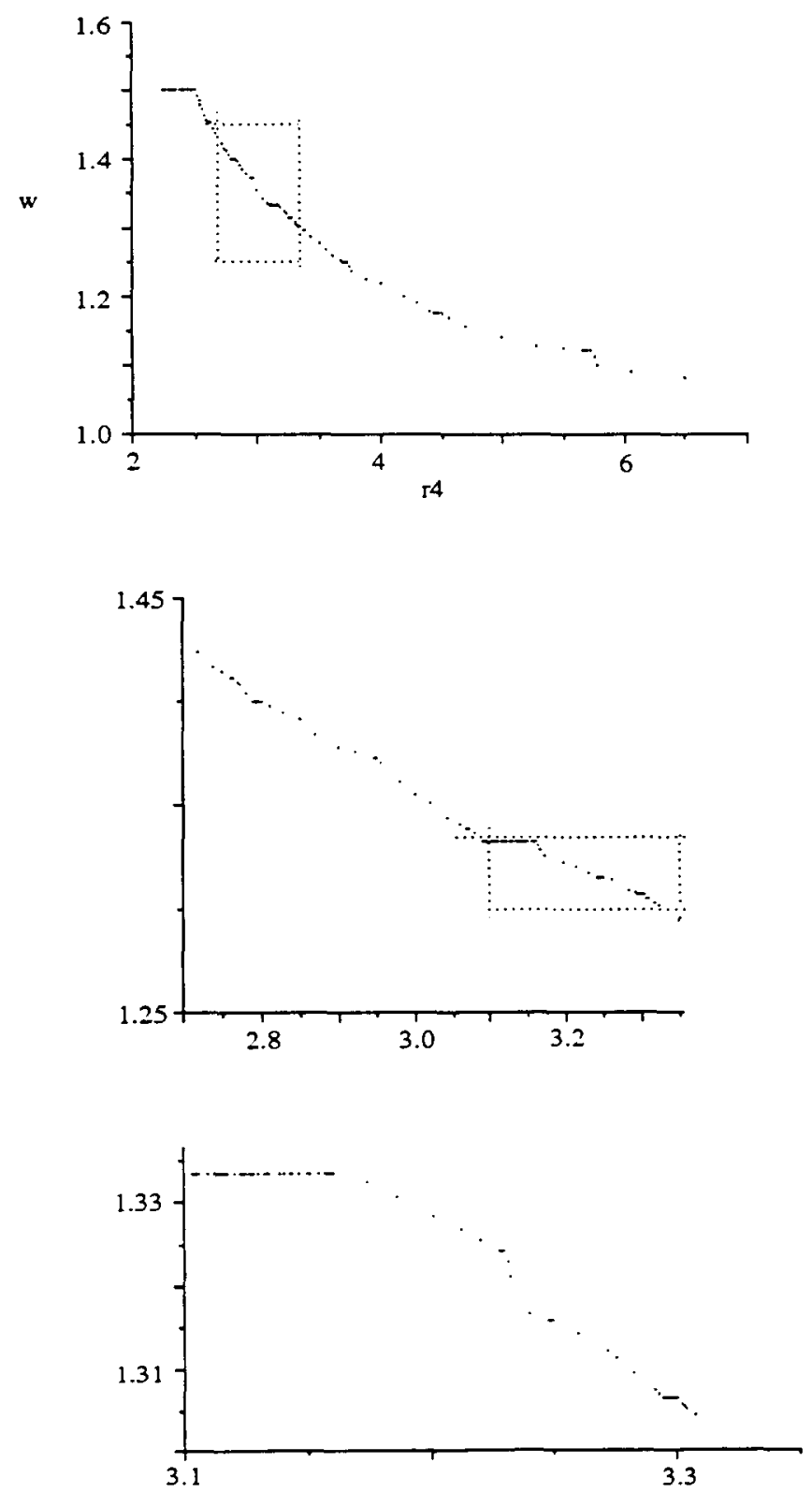

Fig. 2. The "devil's staircase" for the Lotka-Volterra type equations (Eqs. 2a-d, see text). (a) The empirical winding number as a function of the parameter $r_{4}$. The dotted line encloses the part of the figure to be expanded. (b) The range of $r_{4}$ as stipulated by the dotted lines in a. (c) The range of $r_{4}$ as stipulated by the dotted lines in $b$. The fractal nature of the structure is thus revealed by the self similarity evident in comparing a with b with $\mathrm{c}$. 

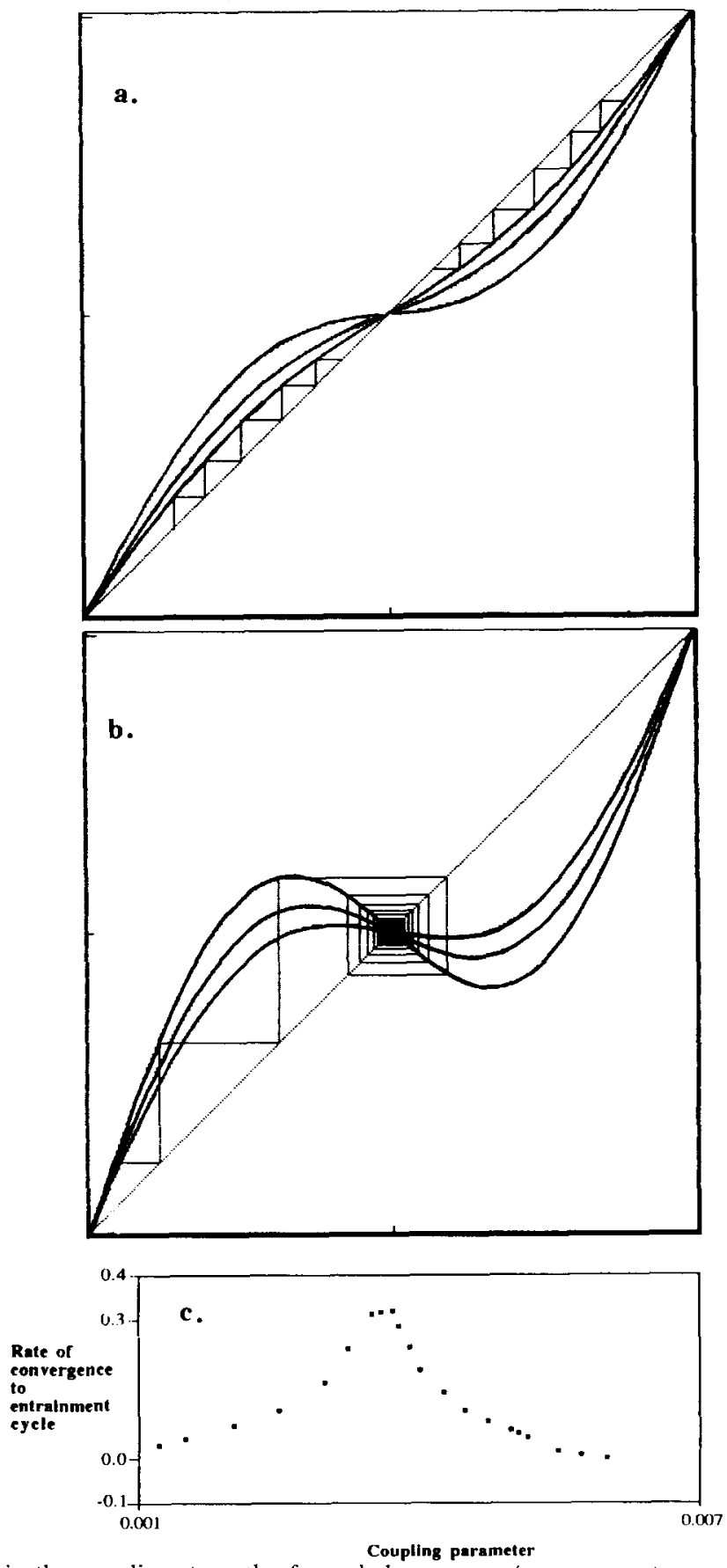

Fig. 3. Changes in the coupling strength of coupled consumer/resource systems as illustrated by the standard circle map. (a) As coupling strength increases from 0 to 0.8 the approach to the stable point is ever more rapid. (b) As coupling strength increases 1 to 1.5 , because of the oscillatory nature introduced at about 0.8 , the approach to the stable point is ever more slowly in this parameter range. (c) Empirical observations of approach to the entrainment cycle of a Lotka-Volterra type system, illustrating the behavior predicted in $a$ and $b$. 


\section{Qualitative results of the non-invertible circle map}

As the parameters $\Omega$ and $k$ of the standard circle map are varied, a well-known pattern emerges in which so-called Arnold tongues defining regions of rational winding numbers increase in area as $k$ increases, as discussed above. The point at which Arnold tongues begin overlapping, usually taken to be at $k=1$, defines the onset of chaos. It is also the point at which the circle map becomes non-invertible. With the exception of a recent analysis of symbolic dynamics (Zeng and Glass, 1989), little attention has been paid to the dynamics of the non-invertible circle map.

A special case exists in which the coupled oscillators are identical, thus making $\Omega=0$. Not only does this case not result in chaos at the non-invertibility point, a classical period doubling approach to chaos can be observed as coupling values reach yet higher values. To see this we begin with a purely uncoupled system for which $\theta(t+1)=\theta(t)$, that is, $k=0$. Increasing $k$ slowly we see the sequence illustrated in Fig. 3. In Fig. 3a, $k$ increases from 0 to 0.8 and the approach to a phase locked position at $\theta=0.5$ is ever more rapid. In Fig. $3 b, k$ increases from 1 to 1.5 , and the rate of approach to the phase locked position is declining. This dynamic corresponds to empirical observations on Lotka-Volterra type coupled oscillators (Vandermeer, 1993) with weak coupling, that the approach to an entrainment cycle is accelerating for low values of coupling, reaches a peak and then becomes decelerating for higher values of coupling, as illustrated in Fig. 3c.

The slope of the map at the non-zero critical point is $s=1-k$, whence the

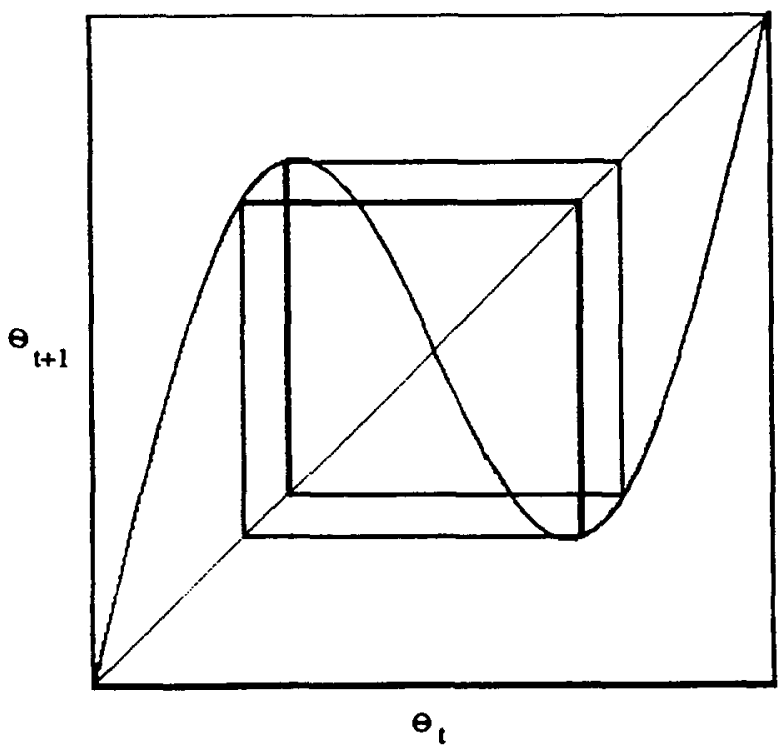

Fig. 4. The qualitative nature of the alternate stable two-point cycles in the non-invertible circle map. 


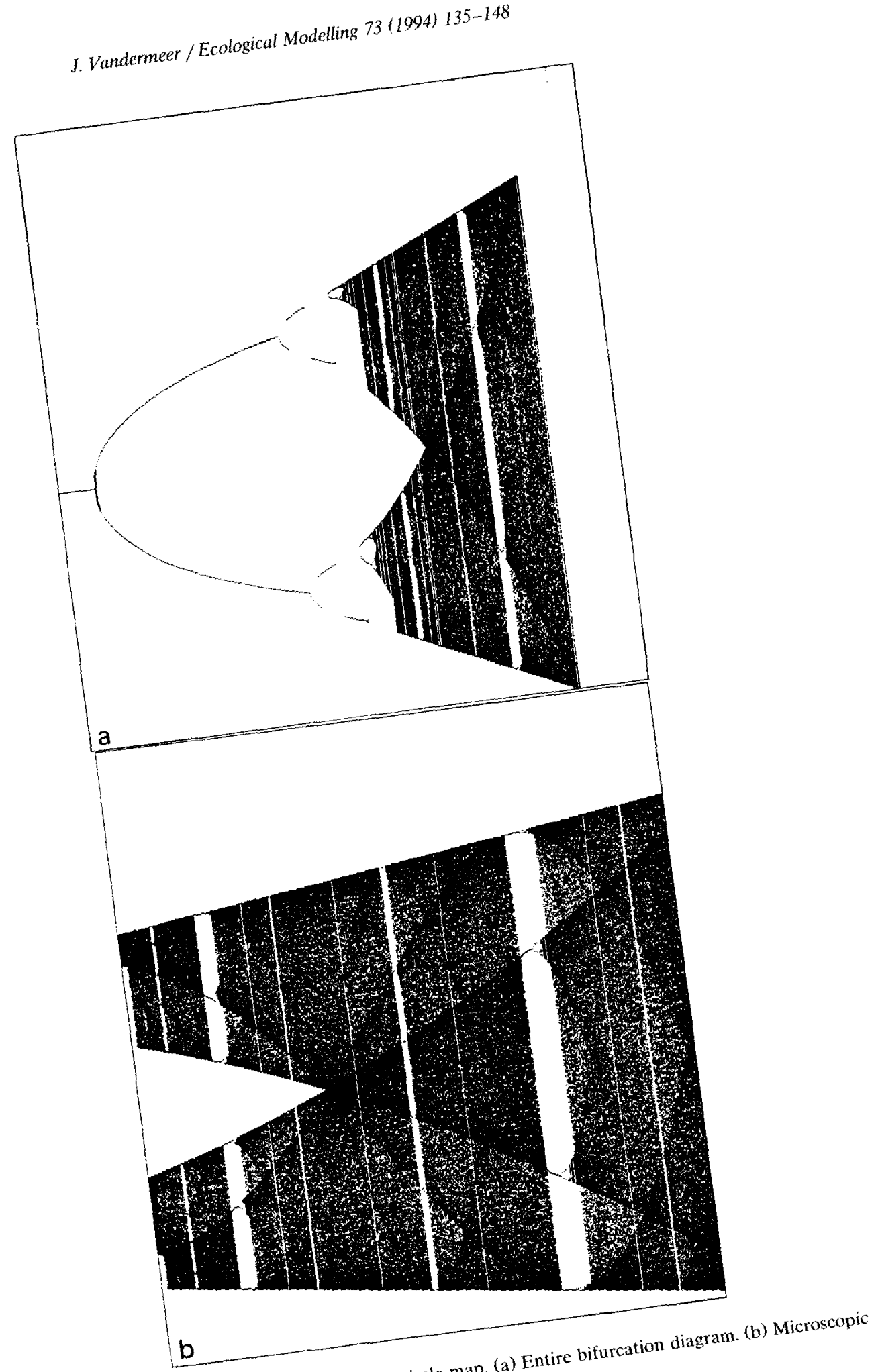

nis Bifurcation diagram for the standard circle map. (indicated with tick marks in a). 
critical point for the first bifurcation (when the slope is exactly -1 ) is simply $k=2$. At the next bifurcation alternative two point cycles begin, as illustrated in Fig. 4 . This bifurcation point is also easily calculated as the point at which the projection from the peak of the sine curve strikes its ascending rather than descending limb. The critical value occurs when the projection from 0.25 strikes exactly at 0.75 , or,
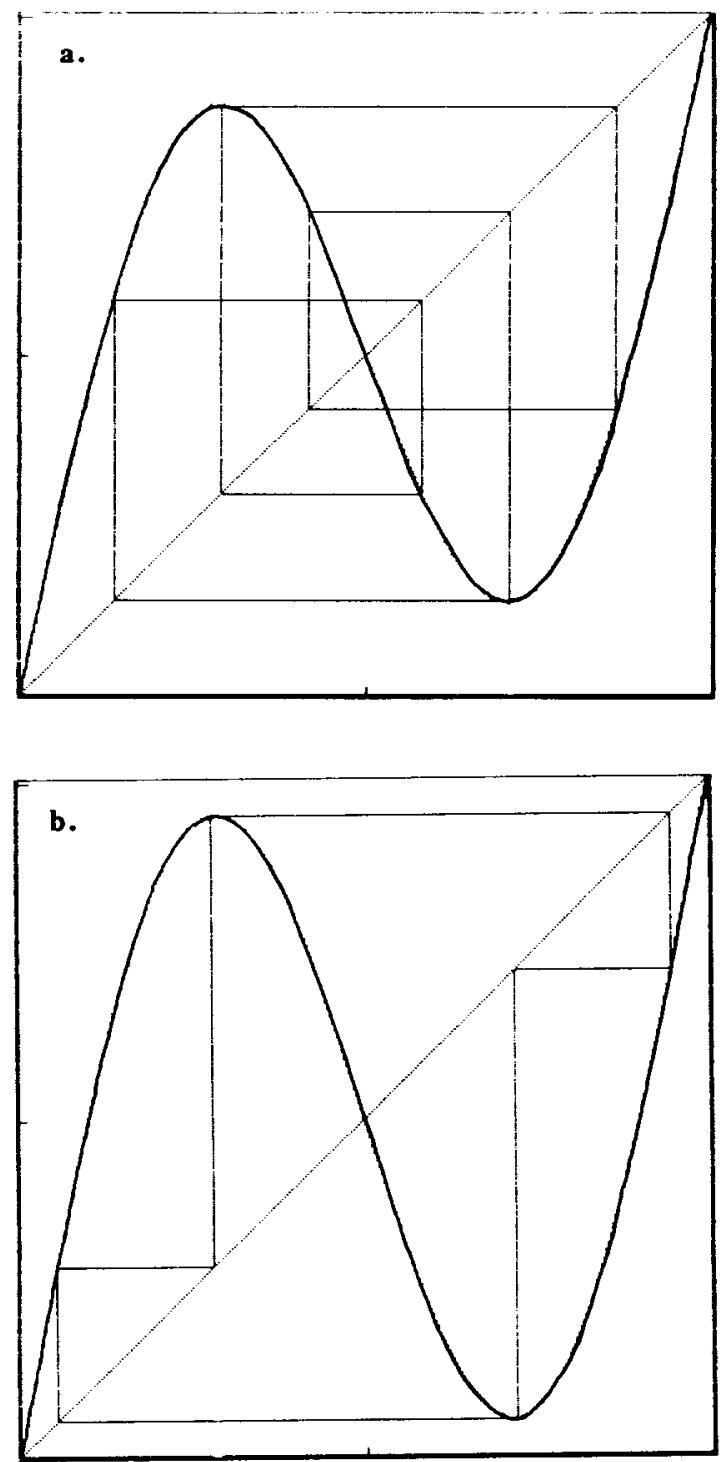

Fig. 6. (a) Illustration of the stable 6-point cycle characterizing the large window on the left hand side of Fig. 5b. (b) Illustration of the stable 4-point cycle characterizing the large window on the right hand side of Fig. $5 b$. 
$0.75=0.25+(k / 2 \pi) \sin (\pi / 2)$, which occurs when $k=\pi$. The next bifurcation marks the point at which both alternate cycles bifurcate to alternative four-point cycles. This sort of partial bifurcation (in the sense that alternate basins always exist) continues until a value of $k$ slightly more than 3.6 , at which point full chaos is apparently reached, and no alternative basins exist.

The entire process can be best visualized with a bifurcation diagram, presented in Fig. 5. As is now familiar, various windows of stable periodicity appear throughout the chaotic region. Two such cases are shown on return maps in Fig. 6, for a period 6 and period 4 window.

The above suggests an approach to chaotic behavior that is quite distinct from that normally associated with the standard circle map, and one that corresponds quite closely to the observations made of coupled biological populations (Vandermeer, 1993).

\section{Discussion}

The discovery that simple biological models can lead to chaos (May, 1976) seems to have left ecology in two alternate modes. On the one hand has been a search for evidence of chaos existing in nature (Schaffer, 1984, 1987; Schaffer and Kot, 1985a, 1986; Schaffer et al., 1988; Hassell et al., 1991), on the other hand a cataloging of ecological situations that have the potential for exhibiting chaos (May, 1976, 1985; May and Oster, 1976; Gilpin, 1979; Shaffer and Kot, 1985b; Allen, 1990; Hastings and Powell, 1991). While we now have a large number of ecological circumstances that clearly imply the potential of chaotic dynamics, and several suggestive cases of chaos in nature, the present offering suggests another avenue for research. If the qualitative results that emerge from a study of circle maps have any significance, we should expect, along with the now commonly expected chaos, demographic locking. Predator-prey pairs coupled together may very well exhibit patterns of correlation in nature that have to do with their demographic locking, thus suggesting the existence of a non-equilibrium pattern.

As discussed elsewhere, one obvious demographic locking pattern is frequency entrainment (Vandermeer, 1993), examples of which are cited frequently in physiology and behavior. The observations of Bulmer (1974) may reflect just such a pattern (Vandermeer, 1993).

Extending the analysis to populations with unlike periodicity, coupling consumer/resource systems suggests that populations will tend to become demographically locked at rational frequencies. Does this then imply that an empirical determination of real periodicities in a natural community will show a clustering around the dominant rational winding numbers? For example, will a community composed of populations roughly obeying Eqs. 2 with the same range of frequencies shown in Fig. 4 have period ratios concentrated near 3/2, 7/5, 4/3, 5/4, and $1 / 1$ as the theory suggests? Of course such a pattern has not yet been sought in any natural community. 
Furthermore, the phenomenon of demographic locking can produce counter-intuitive results that call into question the meaning of both observed correlations and empirical results. For example, in a phase reversed demographic locking of the consumer resource system, Vandermeer and Kaufman (1994a) demonstrated a time series in which two competitors were clearly positively correlated. The phenomenon of demographic locking precludes simplistic expectations of observed correlations of biological phenomena.

The well-known results from circle maps as models of coupled oscillators (Bohr et al., 1984; Bak, 1986) suggest important implications for ecological communities (Vandermeer, 1993). As coupling increases, the parameter $k$ increases, and patterns of phase locking change. Effectively the "steps" in the devil's staircase become broader, eventually overlapping. At this point a sort of competition for different locking frequencies emerges, and the system becomes chaotic. From an ecological point of view, increased coupling implies increased competition between the consumers in the original consumer/resource systems. Thus, the qualitative behavior of the standard circle map implies that as ecological competition increases, the system becomes chaotic. The standard model of interspecific competition that implies coexistence with weak competition and eventual competitive exclusion as competition becomes excessively strong must be modified. As competition proceeds from weak to strong, there is a point at which the competitive system becomes chaotic. The general phenomenon then, must be modified from the classic range of competitive coexistence through competitive exclusion, to a range that extends from stable competitive coexistence, to chaotic competitive coexistence, to competitive exclusion.

Such an ecological interpretation may be important for theories of community structure. While the theory reported here treats only the two-competitor case, it is nevertheless possible to speculate about larger communities. The earlier ideas of MacArthur and Levins (1967) and certain formulations of the equilibrium theory of island biogeography (e.g., MacArthur and Wilson, 1967) suggest that communities tend to a state of full packing, that is, if too many species are in a community, one or more will go extinct, whereas if there are too few, another will colonize. Such a view suggests that communities should be characterized by all species at some maximal allowable level of competition, large enough so that other species cannot invade, yet not too large to cause extinctions. Such a view of communities, in light of the qualitative generalization that strong interspecific competition causes chaos, suggests that populations in natural ecological communities should be characterized by chaotic population behavior.

\section{References}

Abraham, R.H. and Shaw, C.D., 1988. Dynamics - The Geometry of Behavior. Part 4: Bifurcation Behavior. Aerial Press, Santa Cruz, CA, 196 pp.

Abrams, P.A., 1975. Limiting similarity and the form of the competition coefficient. Theor. Popul. Biol., $8: 356-375$. 
Abrams, P.A., 1980. Consumer functional response and competition in consumer-resource systems. Theor. Popul. Biol., 17: 80-102.

Abrams, P.A., 1986. Character displacement and niche shift analyzed using consumer-resource models of competition. Theor. Popul. Biol., 29: 107-160.

Allen, J.C., 1990. Chaos and phase-locking in predator-prey models in relation to the functional response. Fla. Entomol., 73: 100-110.

Arrowsmith, D.K. and Place, C.M., 1990. An Introduction to Dynamical Systems. Cambridge University Press, 423 pp.

Bak, P., 1986. The devils staircase. Phys. Today, December: 39-45.

Bohr, T., Bak, P. and Jensen, M.H., 1984. Transition to chaos by interaction of resonances in dissipative systems. II. Josephson junctions, charge-density waves, and standard maps. Phys. Rev. A, 30: 1970-1981.

Bulmer, M.G., 1974. A statistical analysis of the 10-year cycle in Canada. J. Anim. Ecol., 43: $701-718$.

Chesson, P., 1990. MacArthur's consumer-resource model. Theor. Popul. Biol., 37: 26-38.

Cvitanovic, P., Gunaratne, G.H. and Vinson, M.J., 1990. On the mode-locking universality for critical circle maps. Nonlinearity, 3: 873-885.

Gilpin, M.E., 1979. Spiral chaos in a predator-prey model. Am. Nat., 107: 306-308.

Hassel, M.P., Comins, H.N. and May, R.M., 1991. Spatial structure and chaos in insect population dynamics. Nature, 353: 255-258.

Hastings, A. and Powell, T., 1991. Chaos in a three-species food chain. Ecology, 72: 896-903.

Hsu, S.B. and Hubbell, S.P., 1979. Two predators competing for two prey species: an analysis of MacArthur's model. Math. Biosci., 47: 143-172.

Jackson, E.A., 1989. Perspectives of nonlinear dynamics. Vols I and II. Cambridge University Press, Cambridge, $496 \mathrm{pp}$ and $633 \mathrm{pp}$.

Levine, S., 1976. Competitive interactions in ecosystems. Am. Nat., 110: 903-910.

MacArthur, R.H., 1970. Species packing and competitive equilibria for many species. Theor. Popul. Biol., 1: 1-11.

MacArthur, R.H. and Levins, R., 1967. The limiting similarity, convergence and divergence of coexisting species. Am. Nat., 101: 377-385.

MacArthur, R.H. and Wilson, E.O., 1967. The Theory of Island Biogeography. Princeton University Press, Princeton, NJ.

May, R.M., 1972. Limit cycles in predator-prey communities. Science, 177: 900-902.

May, R.M., 1976. Simple mathematical models with very complicated dynamics. Nature, 261: 459-467.

May, R.M., 1981. Models for two interacting populations. In: R.M. May (Editor), Theoretical Ecology: Principles and Applications. Sinauer, Sunderland, MA, pp. 78-104.

May, R.M., 1985. Regulation of populations with nonoverlapping generations by microparasites: a purely chaotic system. Am. Nat., 125: 573-584.

May, R.M. and Oster, T.F., 1976. Bifurcations and dynamic complexity in simple ecological models. Am. Nat., 110: 573-599.

Schaffer, W.M., 1984. Stretching and folding in lynx fur returns: evidence for a strange attractor in nature? Am. Nat., 124: 789-820.

Schaffer, W.M., 1987. Perceiving order in the chaos of nature. In: M. Boyce (Editor), Evolution of Life Histories: Theory and Pattern from Mammals. Yale University Press, New Haven, pp. 158-178.

Schaffer, W.M. and Kot, M., 1985a. Nearly one dimensional dynamics in an epidemic. J. Theor. Biol., 112: $403-427$.

Schaffer, W.M. and Kot, M., 1985b. Do strange attractors govern ecological systems? BioScience, 35 : $342-350$.

Schaffer, W.M. and Kot, M., 1986. Chaos in ecological systems. Trends Ecol. Evol., 1: 58-63.

Schaffer, W.M., Olsen, L.F., Truty, G.L., Fulmer, S.L. and Graser, D.J., 1988. Periodic and chaotic dynamics in childhood epidemics. In: M. Markus, S. Muller and G. Nicolis (Editors), From Chemical to Biological Organization. Springer, Berlin, pp. 331-347.

Vandermeer, J.H., 1980. Indirect mutualism: variations on a theme by Stephen Levine. Am. Nat., 116: $441-448$. 
Vandermeer, J.H., 1993. Loose coupling of predator prey cycles: entrainment, chaos, and intermittency in the classic MacArthur consumer-resource equations. Am. Nat., 141: 687-716.

Vandermeer, J.H. and Kaufmann, A., 1994a. Why competition implies entrainment and mutualism implies phase reversal: some surprising patterns arising from indirect interactions. J. Theor. Biol. (submitted).

Vandermeer, J.H. and Kaufmann, A., 1994b. Models of coupled population oscillators using 1-D maps. Math. Biosci. (submitted).

Zeng, W.-Z. and Glass, L., 1989. Symbolic dynamics and skeletons of circle maps. Physica D, 40: 218-234. 